\title{
¿Qué hacer ante un pT0 en cáncer de próstata? Revisión a propósito de un caso
}

\section{What to do with a pTO in Prostate Cancer? Review of the Literature and Case Report}

\author{
Milena España Barreiro ${ }^{1}$ Miguel Sebastián Rodriguez Piraquive ${ }^{2}$ Javier Mauricio Salgado Tovar ${ }^{3}$
}

${ }^{1}$ Médica general Universidad del Rosario, Hospital Universitario Mayor - Méderi, Bogotá, Colombia

2 Urólogo Universidad Nacional de Colombia, Hospital El Tunal, Bogotá, Colombia

${ }^{3}$ Urólogo Universidad Nacional de Colombia, Profesor Adscrito Universidad del Rosario, Hospital Universitario Mayor - Méderi, Bogotá, Colombia

Urol Colomb 2019;28:5-8.
Address for correspondence Milena España Barreiro, Médica general Universidad del Rosario, Hospital Universitario Mayor - Méderi, Bogotá, Colombia (e-mail: milenae16@gmail.com).

\section{Resumen}

\section{Palabras Clave}

- cáncer de próstata

- efecto vanishing

- PT0

- prostatectomía radical

- inmunohistoquímica

- resección transuretral

- hormonoterapia

- neoadyuvancia
Introducción y Objetivos El cáncer de próstata es la segunda causa de mortalidad por cáncer en hombres en Colombia y en el mundo. El efecto "vanishing" en cáncer de próstata, es un fenómeno poco frecuente que se define como la ausencia de tumor en el estudio histológico postquirúrgico de pacientes llevados a prostatectomía radical como manejo curativo diagnóstico previo confirmado por biopsia o RTUP. Su incidencia en diferentes series llega hasta el $0,86 \%$, por lo cual existen pocos estudios aleatorizados al respecto y aún no es clara la conducta con ese tipo de tumores. Reportamos un caso de un paciente con cáncer de próstata PT0 y realizamos una revisión de la literatura respecto del seguimiento y manejo que se deberían seguir al enfrentarnos a ese tipo de tumor.

Métodos y Materiales Revisión de la literatura y reporte de caso clínico.

Reporte de Caso Hombre de 58 años sintomático urinario, con PSA elevado por lo que se realizó biopsia transrectal de próstata, con resultado de adenocarcinoma de próstata confirmado por inmunohistoquímica. Al realizarse la prostatectomía radical no se encuentra tumor en la patología. En la literatura se encuentran factores asociados con la presencia del tumor cuando hay RTUP previa y uso de hormonoterapia neoadyuvante en el contexto de tumores de bajo volumen. A pesar de no encontrarse tumor en la patología, está descrita la recaída bioquímica y progresión clínica de esos tumores, por lo que debe realizarse el seguimiento usual para esa patología.

Conclusiones El hallazgo de PT0 en cáncer de próstata, aunque poco frecuente, demanda una guía de manejo Proponemos la revisión de la patología con el protocolo descrito para reducir los falsos positivos y el seguimiento usual con los pasos estandarizados en las guías internacionales. received

February 11, 2018

accepted

April 9, 2018

published online

October 4, 2018
DOI https://doi.org/

10.1055/s-0038-1656558.

ISSN 0120-789X.

eISSN 2027-0119.
Copyright ( 2019, Sociedad Colombiana License terms de Urología. Publicado por Thieme Revinter Publicações Ltda., Rio de Janeiro, Brazil. Todos los derechos reservados.

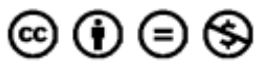




\begin{abstract}
Keywords

- vanishing effect

- radical prostatectomy

- immunohistochemistry

- transurethral resection

- hormone therapy

- neoadjuvant therapy

Introduction and Objectives Prostate cancer is the second leading cause of death related with cancer in men in Colombia and the world. "Vanishing" phenomenon in prostate cáncer is a rare event, where the post-surgical histologic review in patiets that underwent radical prostatectomy is negative, all of them with previous diagnosis of CaP by biopsy or TUR. The incidence of CaP in different series reaches $0.86 \%$, whereby there are few randomized studies and the management and follow up of this type of tumors is not clear. In this article we report a case of a PT0 prostate cancer patient. We perform a systematic review of the literature to determine the medical behavior and medical follow up in cases presenting with PT0 prostate cancer.

Materials and Methods Review of the literature \& case report.

Results Patient of 58 years old with medical history of recent progressive LUTS and high PSA levels that underwent a trans-rectal prostate biopsy with report of adenocarcinoma, that were confirmed by immunohistochemistry. In the pathology report after the radical prostatectomy no tumor cells were found. In the literature, there is reference about factors associated with pT0 prostate cancer in patients with previous RTUP and that were taken to hormonal coadjutant therapy in lesser degree tumors.

Despite the absent of cancer findings in the pathology, it is well described biochemistry relapses and clinical progression of this tumors; usual protocol follow up must be made. Conclusion In patients with PT0 prostate cancer, even though to be a rare finding, must be studied and follow up with the steps mentioned above with the aim to reduce the false positive cases. Also the follow-ups steps must fully meet the requirements of standardized protocols guidelines.
\end{abstract}

\section{Introducción}

El efecto "vanishing” en cáncer de próstata, es un fenómeno poco frecuente que se presenta generalmente en cánceres de bajo volumen tumoral. Incluido dentro de la clasificación del TNM en 2002 como pT0. ${ }^{1}$

Su incidencia en diferentes series varía entre el 0,05\% al $0,86 \%^{2}$ por lo cual existen pocos estudios aleatorizados al respecto y aún no es clara la conducta con ese tipo de tumores.

Reportamos un caso de un paciente con cáncer de próstata pT0 y realizamos una revisión de la literatura respecto del seguimiento y manejo que se deberían seguir al enfrentarnos a ese tipo de tumor.

\section{Método y materiales}

Se realizó una búsqueda en las bases de datos: Science Direct, PubMed y Embase con las palabras clave: Cáncer de próstata; efecto vanishing; PT0; Prostatectomía radical; inmunohistoquímica; resección transuretral; hormonoterapia; neoadyuvancia; Prostate neoplasm; vanishing effect; PT0; radical prostatectomy; immunohistochemistry; transurethral resection; hormone therapy; neoadjuvant therapy. En sus combinaciones con el conector " $Y$ " se encontraron 50 artículos con coincidencias aplicadas al objetivo del trabajo, a los cuales se hizo revisión del resumen, de ellos se descartaron 19 por restricción al acceso de lectura, 5 por fecha de publicación mayor a 15 años, y 6 por un enfoque patológico del manuscrito, posterior a lo cual contamos con 20 artículos de los cuales se descartaron 11 posterior a realizar su lectura por solapamiento de información, bibliografía e ideas centrales. Finalmente, se condesó la información de 9 artículos que resumen la información encontrada en la bibliografía, contando con 2 estudios multicéntricos y ningún reporte de caso.

\section{Reporte de caso}

Paciente de 58 años con historia de 5 años de evolución de síntomas obstructivos del tracto urinario bajo, sin mejoría a pesar de ciclos de tratamiento con Prazosin, Tamsulosina y Dutasteride, progresivos hasta la retención urinaria aguda, la cual fue manejada con sonda uretral a permanencia, y dos intentos de retiro de la misma no exitosos. En seguimiento con tacto rectal y PSA en 9,03 y 7,3 ng/dL en julio y octubre de 2016 respectivamente.

Tacto rectal en dos controles que reportan "próstata adenomatosa, benigna para 35 gramos," valores de PSA descritos, persistentemente elevados (sin asociación a infección de vías urinarias o prostatitis), por lo que se indicó realización de ecografía transrectal de próstata más biopsia. La ecografía reportó una próstata con volumen total de 55 cc, sin áreas hipoecoicas y lóbulo medio intravesical. La biopsia fue leída con un foco de proliferación acinar atípica 
(ASAP) y lesiones sospechosas de corresponder a un adenocarcinoma acinar en el lóbulo izquierdo. Lóbulo derecho negativo para malignidad. Por lo anterior, se complementó con estudios de inmunohistoquímica que confirmaron el diagnóstico de adenocarcinoma de próstata Gleason $3+3$, que comprometía el 5\% del volumen total de uno de los bloques analizados del lóbulo izquierdo. Lóbulo derecho sin compromiso tumoral. Con ese diagnóstico, se ofreció manejo con prostatectomía radical vs radioterapia. En conjunto, se decidió por la primera opción.

El paciente fue llevado en enero de 2017 a prostatectomía radical más linfadenectomía pélvica ampliada.

Inicialmente con buena evolución postoperatoria, con reporte del estudio patológico de la muestra quirúrgica negativo para malignidad.

Se hizo una primera revisión de la muestra por patología, posteriormente se procedió al estudio de la totalidad de la pieza quirúrgica enviada (52 gr), sin tumor residual, se desparafinó cada corte, se reincluyó todo el bloque girando 180 grados y se recortó nuevamente, confirmando que la muestra era negativa para tumor, con evidencia de hiperplasia glanduloestromal, prostatitis crónica y aguda, focos de hiperplasia basaloide y metaplasia escamosa focal.

\section{Discusión}

La primera descripción del efecto "vanishing" se realizó en 1995 en el trabajo de Goldstein y colaboradores, inicialmente se definió como la ausencia de malignidad a la primera revisión microscópica del espécimen quirúrgico, con 13 casos iniciales positivos, que después de la segunda revisión microscópica se redujo a 2 casos definitivamente negativos para malignidad. ${ }^{1,3}$ Se han identificado factores predictores de la presentación de pT0; entre ellos encontramos el bajo volumen tumoral, grado histológico Gleason, mayor tamaño prostático ( $>50-60$ gr) y con una asociación directa, como factores independientes de riesgo, encontramos la hormonoterapia neoadyuvante y la RTUP previa. $^{1,4-7}$

La hormonoterapia neoadyuvante puede disminuir el volumen tumoral al mínimo, logrando así aumentar la incidencia de pT0 de 2,25\% a 15\%, además, también se ha documentado claramente el aumento del riesgo de recurrencia y progresión sistémica en esos pacientes en comparación con los que no recibieron neoadyuvancia. $3,8,9$

Con respecto a la RTUP previa, se puede explicar en bajos volúmenes tumorales por escisión completa del mismo durante la RTUP, aumentando la incidencia de PTO en la patología quirúrgica de $6 \%$ a $25 \%$ en cT1a y del $3 \%$ al $9 \%$ en cT1b. ${ }^{1}$

Bostwick y colaboradores reportaron 38 casos de CaP pT0 de un total de 6843 pacientes llevados a prostatectomía radical entre 1966 y 1995, de los cuales, el $97 \%$ fue diagnosticado por RTUP. ${ }^{1,5,6}$

En un estudio retrospectivo realizado en la Mayo Clinic, se revisaron las patologías de prostatectomías radicales por cáncer realizadas entre 1987 y 2012, del total (22 220) realizadas, se encontró pT0 en 0,3\% (62), cumpliendo los criterios de revisión de la totalidad del espécimen quirúrgico. ${ }^{5,6}$

Mazzucchelli y colaboradores, establecieron un protocolo de revisión para los casos en que la revisión inicial de la patología post quirúrgica fuera negativa que incluye revisión de la biopsia, nueva revisión de las muestras iniciales de la cirugía, desparafinación de las muestras, realización de cortes en niveles adicionales, estudio de inmunohistooquímica y por último, análisis de DNA. Ese protocolo permitió disminuir los falsos positivos de CaP PT0, disminuyendo el diagnóstico final a $1 / 4^{1}$

Por otro lado, Duffield y Epstein, describieron un protocolo similar, que incluye nueva revisión de la biopsia, uso de inmunohistoquímica, en especial en foco sospechoso, para posteriormente, generar nuevos cortes de análisis en bloques con evidencia de neoplasia intraepitelial de alto grado y en la región de la biopsia positiva. Eso va a permitir la confirmación de malignidad del $75 \%$ de los casos inicialmente diagnosticados como pT0. ${ }^{3}$

En el caso de nuestro paciente, se trata de un paciente joven, sin factores de riesgo para

$\mathrm{CaP}$, con una biopsia inicial dudosa, con confirmación de adenocarcinoma de próstata por marcadores inmunohistoquímicos, Gleason $3+3$, con menos del 5\% de volumen tumoral de un sólo bloque, lo que lo clasifica en categoría de riesgo bajo en la clasificación D̀Amico y muy bajo riesgo según los criterios de Epstein. ${ }^{7}$ Fue llevado a manejo quirúrgico, obteniendo una próstata de 52 gramos. Se hizo primera y segunda revisión de la patología, negativa para malignidad, posteriormente se realizaron cortes adicionales, con análisis del volumen total enviado, al cual se le adicionaron estudios de inmunohistoquímica, sin hallazgo de malignidad. Adicionalmente con análisis de vesículas seminales, conductos deferentes y ganglios regionales (\#17) sin evidencia de tumor residual.

Para el CaP PT0, la supervivencia libre de enfermedad a 2 , 5,10 y 15 años después de prostatectomía radical es de $96 \%$, $92 \%, 87 \%$ y $87 \%$ respectivamente, con $1 \%$ de progresión sistémica. ${ }^{6}$ Con respecto al seguimiento de ese tipo de tumores, aunque no existe aún una guía de seguimiento clínico y son pacientes de bajo riesgo, se ha identificado recaída bioquímica hasta en el 11,3\% de los pacientes de todos los grupos de riesgo a 10 años, ${ }^{3,5}$ siendo el principal factor de riesgo la hormonoterapia previa, dentro de los cuales se ha reportado incidencia de hasta el 26\% con menor periodo libre de enfermedad: ${ }^{3}$ por lo que se sugiere un seguimiento como cualquier paciente de bajo riesgo que tuviera patología quirúrgica positiva, cada 3 meses del primer al segundo año, luego semestral y finalmente anual. ${ }^{5,8}$

\section{Responsabilidades éticas}

Protección de personas y animales. Los autores declaran que para esta investigación no se han realizado experimentos en seres humanos ni en animales.

Confidencialidad de los datos. Los autores declaran que han seguido los protocolos de su centro de trabajo sobre la publicación de datos de pacientes. 
Derecho a la privacidad y consentimiento informado. Los autores declaran que en este artículo no aparecen datos de pacientes.

Financiación

Recursos de los autores.

Conflictos de Interés

No se declaran conflictos de interés.

Agradecimientos

Universidad del Rosario, Hospital Universitario Mayor, Méderi.

\section{Bibliografía}

1 Bessède $\mathrm{T}$, Soulié $\mathrm{M}$, Mottet $\mathrm{N}$, et al; Cancerology Committee of the French Urological Association. Stage pT0 after radical prostatectomy with previous positive biopsy sets: a multicenter study. J Urol 2010;183(03):958-962
2 Ebel Sepúlveda L, Corti D, Foneron A, et al. Cáncer evanescente de la próstata. Presentación clínica y revisión histológica. Actas Urol Esp 2009;33(09):956-959

3 Loeb S, Schaeffer EM, Epstein JI. The vanishing prostate cancer phenomenon. Urology 2010;76(03):605-607

4 Vaucher L, Gengler C, Treuthardt C, Jichlinski P. Vanishing prostate cancer: Nightmare for Pathologist or Urologist? Eur Urol Suppl 2007;6(02):181

5 Moreira DM, Gershman B, Boorjian S, et al. Predictors and oncologic outcomes of pT0 prostate cancer in radical prostatectomy specimens. J Urol 2015;193(No. 4S):190-193

6 Moreira DM, Gershman B, Rangel LJ, et al. Evaluation of pT0 prostate cancer in patients undergoing radical prostatectomy. Department of Urology, Mayo Clinic. BJU Int. Doi: 10.1111/bju.13266

7 Bengió RG, Arribillaga L, García Onto H. Evaluación de los criterios de Epstein para cáncer de próstata insignificante en nuestra serie de prostectomía radical. Rev Chil Urol 2012;77(02):77-79

8 Köllermann J, Hopfenmüller W, Caprano J, et al. Prognosis of stage pT0 after prolonged neoadjuvant endocrine therapy of prostate cancer: a matched-pair analysis. Eur Urol 2004;45(01):42-45

9 Hammerer P. pT0 after radical prostatectomy: overtreatment for insignificant prostate cancer? Eur Urol 2004;45(01):35 